\title{
CARACTERIZACIÓN DERMATOGLíFICA DE LAS CICLISTAS COLOMBIANAS DE PISTA DE ALTOS LOGROS EN PRUEBAS DE SEMIFONDO
}

\section{DERMATOGLYPHIC CHARACTERISTICS OF FEMALE COLOMBIAN CYCLISTS OF HIGH ACHIEVEMENT IN UNDERCARD TRY-OUTS}

\author{
Juan Pablo Medellín ${ }^{1}$ \\ ${ }^{1}$ Profesional en Ciencias del Deporte, Universidad de Ciencias Aplicadas y Ambientales U.D.C.A, Bogotá, D.C., e-mail: \\ m_juanpablo9@hotmail.com.
}

Rev. U.D.C.A Act. \& Div. Cient. 17(1): 45-52, 2014

\section{RESUMEN}

Las características dermatoglíficas son actualmente empleadas a nivel mundial, para determinar y seleccionar los talentos en las más variadas disciplinas deportivas. Para encontrar el perfil genético positivo que puede ser aplicado para este objetivo, se analizan las características de deportistas sobresalientes en el deporte específico. Con el objetivo de generar un perfil preliminar dermatoglífico que sirva para la selección de talentos en el ciclismo de pista femenino, en pruebas de semifondo, se evaluaron atletas de selección Colombia y medallistas en Juegos Nacionales. La colecta de dermatoglifos, se realizó, previa firma de consentimiento, en una muestra de seis ciclistas, cuya edad osciló entre 22,3 \pm 4 años. El estudio fue descriptivo y evaluó las variables: arco, presilla, verticilio, D10 y cantidad total de líneas (STCL). Los resultados, se presentan en un radar con los valores normalizados, en el cual, se detecta que los valores más destacados corresponden a la predominancia de dibujos presilla y verticilos, disminución de arcos y un D10 y SCTL medio.

Palabras clave: Huellas, genética, talento, ciclismo.

\section{SUMMARY}

Dermatoglyphic characteristics are currently used worldwide to identify and select the most diverse talents in sports. To find the positive genetic profile that can be applied for this purpose, it analyzes the characteristics of outstanding athletes in specific sports. In order to generate a preliminary dermatoglyphic profile to serve for the talent selection in women's track cycling, in undercard tests a Colombian team and national games medalists were evaluates. The dermatoglyphics collecting was by previously signed consent, in a sample of six cyclists, whose age was $22.3 \pm 4$ years. The study was descriptive and variables evaluated: arch, loop, whorl, D10 and number total of lines (STCL). The results are presented in the normalized radar values, which detect most outstanding values, corresponding to the predominance of loop and whorls designs, decreased arches, and D10 and SCTL medium.

Key words: Footprints, genetic, talent, cycling.

\section{INTRODUCCIÓN}

La trayectoria de los ciclistas colombianos, en la modalidad del ciclismo de pista, se ve reflejada en logros de alto nivel, dentro del último lustro; entre los triunfos más destacados encontramos medallas de oro en juegos panamericanos, suramericanos, centroamericanos y del Caribe y campeonatos mundiales, en diversidad de pruebas, dentro de las que se destacan, el omnium, la madison, la persecución por equipos e individual y la prueba por puntos dentro del semifondo, lo que les ha valido la aparición en los Juegos Olímpicos de Londres 2012; la tabla 1 (UCI, 2013) relaciona las posiciones de Colombia dentro de las pruebas de semifondo, en rama femenina, categoría mayores.

Dado el origen multifactorial de los éxitos deportivos (Borin \& Gonçalves, 2004a; Vona et al. 2005), que se derivan de la interacción de muchos componentes que actúan sinérgicamente (Rankinen et al. 2006; Borin \& Gonçalves, 2004b), se hace necesario determinar cuáles son los componentes que conforman a los campeones deportivos, en cada modalidad existente.

Una de las condiciones necesarias para lograr el éxito en el alto rendimiento es el talento innato o la idoneidad del 
Tabla 1. Ranking de Colombia dentro del ciclismo de pista en pruebas de semifondo, en la rama femenina.

\begin{tabular}{|c|c|c|c|}
\hline CATEGORÍA & COMPETENCIA & POSICIÓN & PUNTOS \\
\hline \multirow{4}{*}{ MAYORES } & TEAM PURSUIT & 15 & 450 \\
\cline { 2 - 4 } & OMNIUM & 47 & 85 \\
\cline { 2 - 4 } & POINTS RACE & 29 & 105 \\
\cline { 2 - 4 } & 3km INDIVIDUAL PURSUIT & 6 & 220 \\
\cline { 2 - 4 } & SCRATCH & 29 & 105 \\
\hline
\end{tabular}

Adaptado de: UIC - Unión Ciclística Internacional (2013).

atleta (Dos Santos et al. 2007). Según Harris (1982), citado en el manual del Programa de Oportunidades de Talentos (TOP) de Gimnasia EE.UU (1998), el desarrollo de los atletas jóvenes se puede mejorar si se entrena para el deporte, en el cual, se posee el mayor potencial. Weineck (1999) define como un atleta talentoso a alguien con voluntad y con disposición para el rendimiento y está por encima del promedio para ese grupo de edad; al comprobarse su desempeño superior en competencia y el componente biológico es determinante para detectar talentos deportivos, lo que permite dilucidar sujetos predispuestos a determinadas disciplinas (Zakharov, 2003).

El desarrollo y el perfeccionamiento de las habilidades motoras y sus estándares son influenciados de manera compleja (Gallahue \& Ozmun, 2005); la eficiencia del sistema neuromotor dependerá, fundamentalmente, de la predisposición genética de la persona (Dantas, 2003). Este concepto es reforzado por Norton \& Olds (2001), quienes indican que, en caso de existir un tipo de cuerpo ideal para un deporte en particular, sólo los atletas con esta forma ideal podrán permanecer a nivel competitivo.

Es necesario destacar que cada persona es única en el proceso de desarrollo y responde individualmente al desafío del cambio y evolución a un nivel determinado por condiciones genéticas, ambientales y biológicas, en combinación con las necesidades específicas de la función motora (Fraga de Oliveira \& Fernandes, 2008).

Conocer las características de un deporte parece ser la correcta aplicación de las estrategias que influyen en su mejor desempeño; para actuar de acuerdo con la competencia, un atleta debe reunir las características físicas, antropométricas, fisiológicas y psicológicas específicas exigidas por la modalidad, lo cual, permitirá entender las manifestaciones fenotípicas, como resultado de la influencia del medio sobre el genotipo (Junior et al. 2006). Una combinación de características favorables al deporte hará que se clasifique como un "talento deportivo" (Ferreira \& Fernandes Filho, 2003). El fenotipo puede diagnosticar muchas variables de una injerencia directa en deporte de alto nivel; sin embargo, estas variables son manipulables, por ello, el genotipo cobra mayor importancia, dado que no es posible su manipulación, excepto cuando hubo cambios ilegales para mejorar el rendimiento (dopaje) (Ferreira \& Fernandes Filho, 2003).

La apariencia fenotípica puede influir y disparar los posibles cambios en las características genotípicas en un determinado deporte (Dantas et al. 2004). Desde la perspectiva de Del Villar (1992), la constitución física es el resultado de ciertos parámetros hereditarios genéticos, que pueden ser el objetivo para el desarrollo del ejercicio físico. El progreso de un atleta es el resultado de la influencia mutua de la herencia y del ambiente, cuando el objetivo es identificar las habilidades motoras características de la modalidad y determinar el grado de influencia de factores hereditarios y ambientales en cada una (Dantas et al. 2004), dándose un proceso de planificación estratégica, de cara a la carrera deportiva del atleta.

La influencia de factores genéticos y ambientales no son los mismos en los diferentes períodos de edad, ya que existen momentos especiales, sometiendo a consideración la sensibilidad a diversos factores ambientales, de aprendizaje y de entrenamiento y es allí donde se observa una disminución en el nivel genético y una creciente dependencia de los factores ambientales (Dantas et al. 2004). Los periodos sensibles (edad, duración) son apropiados para la edad biológica y no para la cronológica. Está demostrado que el desarrollo de las habilidades motoras o el carácter de la adaptación del organismo de los adolescentes de 12 a 16 años de edad no depende sólo de la edad cronológica, pero sí de los ritmos individuales de desarrollo (Volkov, 1983).

La capacidad de diagnosticar y de comprender el potencial de genotipo de cada atleta, junto con la contribución fenotípica puede mejorar la selección y la formación de nuevos individuos en la alta cualificación (Ferreira \& Fernandes Fil- 
ho, 2007; João \& Fernandes, 2002; Skinner, 2002; Dos Santos et al. 2007; Maia, 1999). Por ello, la creación de nuevos modelos de caracterización, fundamentados en la ciencia y la predicción del rendimiento, ayudarán a la selección y a la orientación de talentos deportivos (Weineck, 1991; Filin \& Volkov, 1998; Bohme, 1994; Fonseca et al. 2008), los cuales, estarían en la capacidad de tolerar grandes cargas de entrenamiento acompañado de grandes índices de mejora deportiva (Filin \& Volkov, 1998). Este modelo, se debe basar en el perfil genético, funcional, psicológico y somatotípico de atletas de alto rendimiento de cada deporte (Filin \& Volkov, 1998; Fonseca et al. 2008) y debería ser elaborado según los datos y los índices de la población a la que pertenecen los individuos. Fernandes Filho (1999) y João et al. (1999) juzgan que configurar el perfil para un grupo en el que se desee intervenir puede ser la diferencia entre el éxito y el fracaso de la estrategia, en la programación del entrenamiento deportivo.

El modelo del deportista de alto nivel debe determinar no solamente las características más sustanciales de la modalidad (características del modelo), sino también los posibles parámetros de desviación del "ideal", presentándose como innatos al individuo, así como para estipular ciertos cambios en el inicio del desarrollo, que suponen la aptitud deportiva (Junior et al. 2006; Neto \& Baptista, 2007).

Es de gran importancia recalcar que la observación y la determinación de los parámetros óptimos para todas las disciplinas deportivas, a través de un perfil de características comunes, no es un estereotipo de exclusión, es por el contrario, un esfuerzo de satisfacer las demandas de cada deporte con sus peculiaridades. Ser un atleta no es una tendencia común para todas las personas, es una minoría. Además, dentro de ésta, el factor de oportunidad juega un papel clave (Dantas et al. 2004; Dantas \& Fernandes, 2002; Ferreira \& Fernandes Filho, 2003), pues contribuye a la concepción teórica general sobre la especificidad de la constitución del hombre, de acuerdo con el perfil de su actividad (Castanhede et al. 2003; Junior et al. 2006).

De esta manera, se puede conseguir no sólo el rendimiento deportivo máximo, sino también la dirección correcta de esfuerzos, de tiempo y de dinero (Ribeiro Ferreira et al. 2008). La intención de apreciar el rendimiento y comprobar el perfil de los atletas en la fase inicial, con el objetivo de obtener un alto rendimiento, a través de las respuestas del fenotipo y del genotipo, son necesarios para lograr el éxito, de acuerdo con Ferreira \& Fernandes Filho (2003).

El uso de la dermatoglífia en la selección y en la orientación de talentos deportivos fue impulsado por investigadores rusos, quienes determinaron la relación entre indicadores dermatoglíficos y cualidades físicas, influyentes dentro del rendimiento deportivo (Leiva et al. 2011).

\section{MATERIALES Y MÉTODOS}

Para esta investigación, se siguieron las reglas ordenadas por el Ministerio de Salud, para trabajos con seres humanos o con muestras de origen humano (Resolución N $N^{\circ} 008430$ de 1993, Resolución No. 01 de 2008. Ministerio de Salud).

La muestra de individuos estuvo constituida por ciclistas colombianas de pista, especializadas en las pruebas de semifondo: medallistas en Juegos Nacionales 2012 (5), integrantes de la Selección Colombia; participante en el Panamericano de Ciclismo de Pista (1), realizado en Aguascalientes, México; Copa Mundo de Pista (2), que tuvo lugar en Cali, Colombia; Campeonato Mundial Juvenil de Pista (1), llevado a cabo en Invercagill, Nueva Zelanda y los XVII Juegos Deportivos Bolivarianos Trujillo 2013 (3).

Para verificar las características dermatoglíficas, se utilizó el protocolo propuesto Cummins \& Midlo (1963). La recolección de impresiones digitales, se realizó cubriendo de tinta negra las falanges distales y haciendo una rodada uniforme en las planillas diseñadas para este proceso.

Posteriormente, a la realización del dibujo dactilar, se clasificó y se efectuó el conteo de crestas; así se pudo determinar la predisposición del sujeto para la realización de una modalidad deportiva, en especial (Cummins \& Midlo, 1963), analizando variables cualitativas (diseños) y cuantitativas (SCTL, D10):

a) Los diseños, arcos, presillas y verticilios (Figura 1) de las falanges dístales de ambas manos, la cantidad de diseños en los dedos de las manos derecha e izquierda y la complejidad en los diseños de los diez dedos de las manos (D10), calculada por la ecuación:

$$
\mathrm{D} 10=\Sigma \mathrm{L}+2 \sum \mathrm{W}
$$

Dónde: Presillas (L); Verticilos (W)

b) Cantidad de líneas. Se cuenta cada cresta que cruza o toca la línea imaginaria (Línea de Galton), trazada desde el delta hasta el núcleo, sin incluir la cuenta del delta o del núcleo; con base en la cantidad de líneas de todos los dedos de las manos, se calcula SCTL, que es la sumatoria de la cantidad de líneas de los dedos de las dos manos (Dantas, 2012; Castanhede et al. 2003).

La gráfica 1 corresponde a la normalización de los resultados, donde los límites se hallaron con las siguientes ecuaciones: $=($ Dato - Min. $) /($ Max. - Min. $)$

Y Finalmente, se normalizan los límites:

Lim. Inf. $=X-(1,96 * S) / \mathrm{n}^{\wedge}(1 / 2)$

Lim. Sup. $=X+(1,96 * S) / \mathrm{n}^{\wedge}(1 / 2)$ 

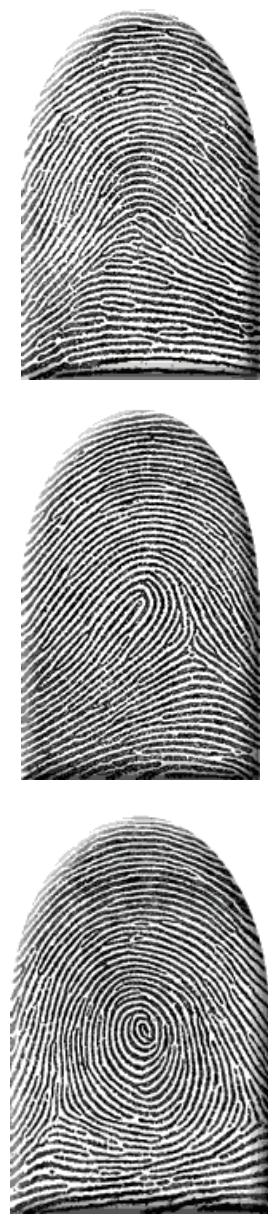

Arco

Se clasifica con la letra A, es el primer tipo del sistema, está formado por líneas papilares más o menos paralelas a la base del pliegue de flexión de la tercera falange, que atraviesan el dactilograma de un extremo a otro y carece de deltas, líneas directrices y núcleo.

\section{Presilla}

Se clasifican con la letra L, está formada por un delta que se encuentra ubicado a la derecha del observador y sus líneas directrices que envuelven a las nucleares se dirigen hacia la izquierda.

\section{Verticilo}

Se clasifica con la letra $\mathrm{V}$, es el último tipo del sistema, está formado por dos deltas, uno a la derecha y el otro a la izquierda y sus líneas directrices circunscriben al núcleo en diferentes formas.

Figura 1. Impresiones dactilares según su dibujo arcos, presillas y verticilos. (Tomado de Guzmán, 2003; Fonseca et al. 2008; Martínez et al. 2012; Abramova et al. 1996; Nishioka et al. 2007; João \& Fernandes Filho, 2002).

\section{RESULTADOS Y DISCUSIÓN}

Las impresiones dactilares se dividen según su dibujo en tres grandes grupos: arcos, presillas y verticilos, como lo muestran Avella \& Medellín (2013), quienes adaptaron las figuras de Guzmán (2003); Fonseca et al. (2008); Martínez et al. (2012); Abramova et al. (1996); Nishioka et al. (2007) y João \& Fernandes Filho (2002) (Figura 1). Los dibujos dactilares pueden ser divididos en subgrupos, con base a diferencias menores que existen entre ellos.

La edad de las ciclistas y las características dermatoglíficas recogidas durante el presente estudio, se muestran en la tabla 2.
Tabla 2. Resultados estadísticos de las variables analizadas.

\begin{tabular}{|l|c|c|}
\hline \multirow{2}{*}{} & \multicolumn{2}{|c|}{ Ciclistas $(\mathbf{n}=\mathbf{6})$} \\
\cline { 2 - 3 } & Media & Desviación \\
\hline Arco & 2 & 1,897 \\
\hline Presilla & 4 & 3,162 \\
\hline Verticilo & 4 & 4,382 \\
\hline D10 & 12 & 5,967 \\
\hline SCTL & 115,5 & 76,943 \\
\hline
\end{tabular}




\section{Valores Normalizados}

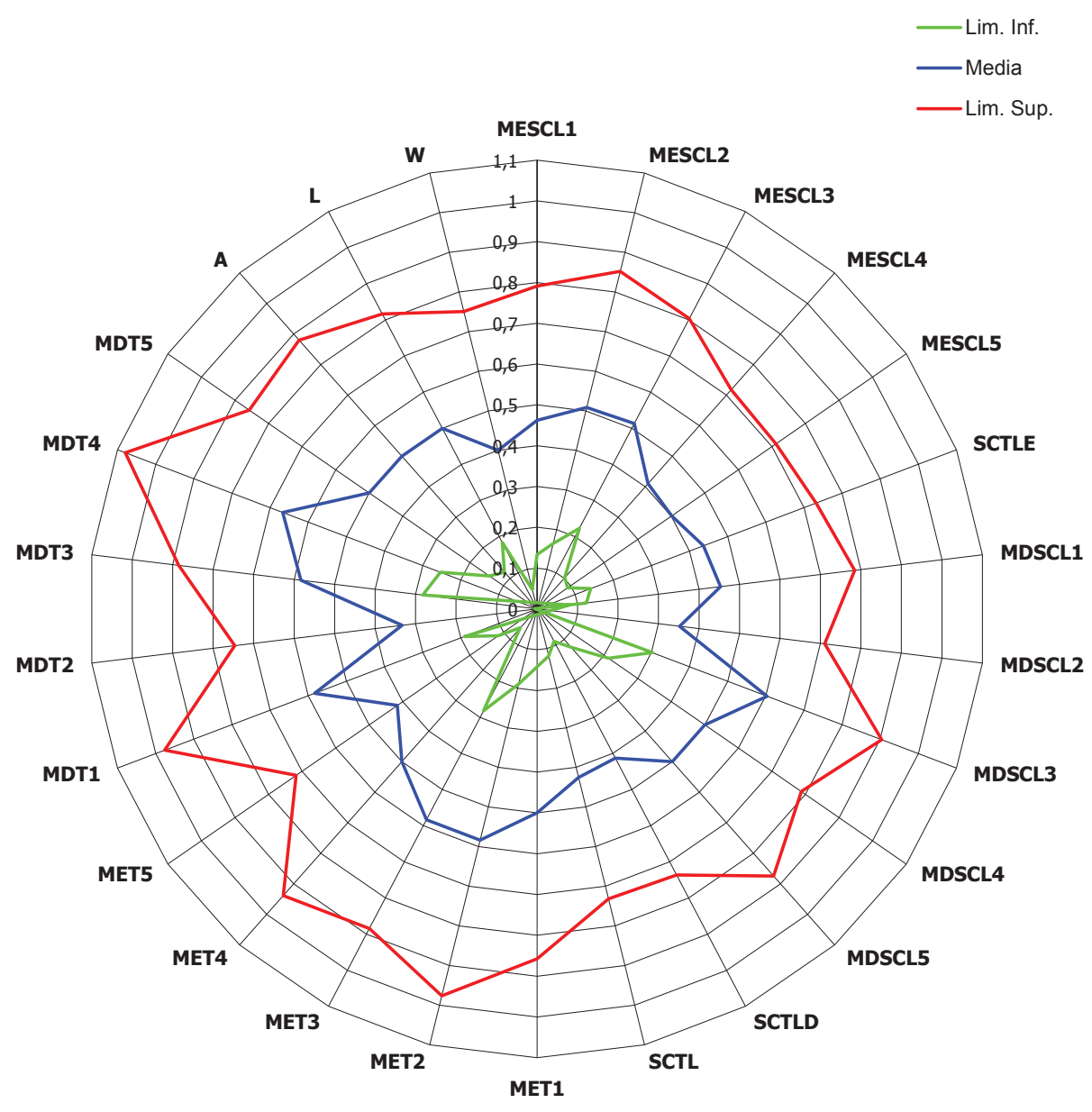

Gráfica 1. Radar con los valores normalizados.

MESCL = Mano Izquierda Sumatoria Líneas; $M D S C L=$ Mano Derecha Sumatoria Líneas; SCTLE $=$ Sumatoria Total Líneas Mano Izquierda; SCTLD = Sumatoria Total Líneas Mano Derecha; SCTL= Sumatoria Cantidad Total de Líneas; MET= Deltas Mano Izquierda; $M D T=$ Deltas Mano Derecha; $\mathrm{L}=$ Presilla; $\mathrm{W}=$ Verticilo; $\mathrm{A}=$ Arco.

Si se comparan estos resultados con aquellos obtenidos por Tuche et al. (2005) con ciclistas varones de ruta brasileños (Tablas 3 y 4), se observa cierta similitud en el bajo porcentaje de aparición de Arcos en los deportistas. Asimismo, el promedio del D10 y el SCTL fueron prácticamente idénticos; sin embargo, la desviación de las variables mencionadas en las ciclistas colombianas es superior a los brasileños. Este resultado se podría atribuir al tamaño de la muestra o a que la muestra del presente estudio exhibe diferencias entre las tres ciclistas Selección Colombia, actuales asistentes a los Juegos Bolivarianos de Trujillo 2013 y el resto de las deportistas; las convocadas son en las variables cualitativas más similares a los datos de Tuche et al. (2005) y, cuantitativamente hablando, los datos de las seleccionadas por Colombia son menores a los ciclistas varones, lo que podría responder a las diferencias entre modalidades deportivas. 
Tabla 3. Comparación variables cualitativas.

\begin{tabular}{|l|c|c|}
\hline \multirow{2}{*}{} & Colombia $(\mathbf{n}=\mathbf{6})$ & Brasil $(\mathbf{n}=\mathbf{1 8})$ \\
\cline { 2 - 3 } & Media & Media \\
\hline Arco & $20 \%$ & $10 \%$ \\
\hline Presilla & $40 \%$ & $62 \%$ \\
\hline Verticilo & $40 \%$ & $28 \%$ \\
\hline
\end{tabular}

Tabla 4. Comparación variables cuantitativas.

\begin{tabular}{|l|c|c|c|c|}
\hline & $\begin{array}{c}\text { Colombia Pista } \\
\text { Damas }(\mathbf{n}=\mathbf{6})\end{array}$ & $\begin{array}{c}\text { Brasil Ruta Varones } \\
\mathbf{( n = 1 8 )}\end{array}$ & $\begin{array}{c}\text { Colombia Pista } \\
\text { Damas } \mathbf{( n = 6 )}\end{array}$ & $\begin{array}{c}\text { Brasil Ruta Varones } \\
\mathbf{( n = 1 8 )}\end{array}$ \\
\cline { 2 - 5 } & Media & Media & Desviación & Desviación \\
\hline D10 & 12 & 11,8 & 5,967 & 3,43 \\
\hline SCTL & 115,5 & 114,8 & 76,943 & 38,21 \\
\hline
\end{tabular}

La comparación intragrupo demostró que la mitad de más alto rendimiento de la muestra (dos ciclistas participantes actualmente en Selección Colombia y la reciente campeona mundial juvenil del scratch) presenta similitudes significativas en sus variables cuantitativas: SCTLE: $40,7 \pm 5,5$; SCTLD: 42,3 \pm 19,3; SCTL: $83 \pm 24,8$; D10: $9 \pm 1$, lo que explicaría su homogeneidad en resultados y logros.

Una comparación entre las atletas velocistas colombianas (Avella \& Medellín, 2013) con la muestra del presente estudio arroja una similitud en las variables de sumatoria de líneas por dedo en ambas manos (MDSCL - MESCL), a excepción del quinto dedo de la mano izquierda. Se presentan variables oscilantes entre 0,7 y 0,9 y, por ende, se evidencia un gran parecido en la sumatoria total de líneas por mano (SCTLD - SCTLE), esta con más énfasis en la mano derecha. Ésto se justifica por Leiva et al (2011), quien afirma que la sumatoria de líneas en la mano derecha está asociada a las capacidades de velocidad y de fuerza, mientras que la misma sumatoria en la mano izquierda vincula la resistencia y la coordinación motora. Así, aunque la presente muestra se especializa en pruebas de semifondo, su naturaleza real es mixta, en la cual, ambas vías metabólicas son partícipes; por ello es razonable una similitud y una predisposición genética a esfuerzos sub máximos. En cuanto a las diferencias marcadas está la enorme superioridad en dibujos presilla de las atletas contra las ciclistas, que es casi el doble, dado que su naturaleza deportiva demanda una tendencia importante a actividades alácticas, lo que difiere en parte a las ciclistas.

De la normalización y del análisis de los datos por individuo, se generan la gráfica 1 , que permite tener un panorama grupal sobre las condiciones dermatoglíficas y establece un modelo gráfico preliminar, para orientar el proceso en la selección de talentos.

Se sugiere realizar estudios en deportistas de alto nivel con la misma orientación de esfuerzo, en este caso, deportes de semifondo nutrirían esta base de datos y harían más significativo el perfil a construir. Asimismo, el procedimiento se debería replicar en todos los deportes y agruparlos según su vía metabólica predominante.

Agradecimientos: A Andrés Felipe Bohórquez Aldana, por alentar el inicio de este proyecto y por servir de soporte para familiarizarme con la dermatoglífia. Al Profesor José Fernandes Filho Ph.D., por su apoyo en la normalización de los datos y la construcción de los radares; a la Universidad de Ciencias Aplicadas y Ambientales U.D.C.A, por el apoyo financiero para la realización de la investigación y a todos los Ciclistas que permitieron la toma de sus datos para la construcción de este producto. Conflicto de intereses: El manuscrito fue preparado y revisado con la participación del autor y declaro que no existe ningún conflicto de intereses que ponga en riesgo la validez de los resultados.

\section{BIBLIOGRAFÍA}

1. ABRAMOVA, T.; NIKITINA, T.; OZOLIN, H. 1996. Posibilidades del empleo de la dermatoglífia dactilar en la selección deportiva. Teoría y práctica de la cultura física. 3:8-14. 
2. AVELLA, R.E.; MEDELLÍN, J.P. 2013. Perfil Dermatoglífico y Somatotípico de la Selección Colombia de Atletismo (Velocidad) participante en los Juegos Panamericanos de Guadalajara, 2011. Rev. U.D.C.A Act \& Div. Cient. 16(1):17-25.

3. BOHME, M. 1994. Talento esportivo I: aspectos teóricos. São Paulo: Rev. Paulista Ed. Fís. 8(2):90-100.

4. BORIN, J.; GONÇALVES, A. 2004a. Alto nível de rendimento: a problemática do desempenho esportivo. Rev. Bras.Cienc. Esporte. 26(1):9-17.

5. BORIN, J.; GONÇALVES, A. 2004b. Saúde coletiva e atividade física e talento desportivo. In: GONÇALVES, A. (org). Conhecendo e discutindo saúde coletiva e atividade física. Rio de Janeiro: Guanabara Koogan. p.61-81.

6. CASTANHEDE, A.; DANTAS, E.; FERNANDES, FILHO, J. 2003. Perfil dermatoglífico e somatotípico, de atletas de futebol de campo masculino, do alto rendimento no Rio de Janeiro - Brasil. Fitness \& Perform. J. 2(4):234-239.

7. CUMMINS, H.; MIDLO, C.H. 1963. Finger prints, palms and soles. An introduction to dermatoglyphics. Dover Publications. 319p.

8. DANTAS, E. 2003. A Prática da Preparação Física. $5^{\mathrm{a}}$ ed. Rio de Janeiro: Shape. p.200-220.

9. DANTAS, E. 2012. La práctica de la preparación física. Editorial Paidotribo. Barcelona. p.227-383.

10. DANTAS, E.; FERNANDES FILHO, J. 2002. Identificação dos perfis genético, de aptidão física e somatotípico que caracterizam atletas masculinos de alto rendimento, participantes do futsal adulto no Brasil. Fitness \& Perform. J. 1(1):28-36.

11. DANTAS, P.; ALONSO, L.; FERNANDES FILHO, J. 2004. A Dermatoglífia no Alto Rendimento do Futsal Brasileiro. Fitness \& Performance Journal, v.3, n.3, p. $136-142$.

12. DEL VILLAR, C. 1992. La preparación física del futbol basada en el atletismo. Madrid: Gymnos. p.12-20.

13. DOS SANTOS, L.; SILVA DANTAS, P.; FERNANDES FILHO, J. 2007. Características Genotípicas e Fenotípicas em Atletas Velocistas. Rev. Desporto e Saúde da Fundação Técnica e Científica do Desporto. 4(1):4956.
14. FERNANDES FILHO, J. 1999. A prática da avaliação física. Rio de Janeiro: Shape. p.6-10.

15. FERREIRA, A.A.M.; FERNANDES FILHO, J. 2003. Corrida de Orientação: Caracterização dermatoglifica e somatotípica de alto rendimento da região sul do Brasil. Fitness \& Performance J. 2(3):145-150.

16. FERREIRA, H.F.; FERNANDES FILHO, J. 2007. Diagnostico da predominância do tipo de fibra muscular da seleção brasileira de canoagem slalom através da Dermatoglifia. FIEP Bull. 77:273-275.

17. FILIN, V.; VOLKOV, V. 1998. Seleção de Talentos nos esportes. Londrina: Miograf. p.10-40.

18. FONSECA, C.; DANTAS, E.; FERNANDES, P.; FERNANDES FILHO, J. 2008. Perfil dermatoglífico, somatotípico e da força explosiva de atletas da seleção brasileira de voleibol feminino. Fitness \& Performance $J$. 7(1):35-40.

19. FRAGA DE OLIVEIRA, E.; FERNANDES FILHO, J. 2008. Behavior of genotype and phenotype characteristics in children practicing and not practicing swimming. FIEP Bulletin V. 78 Special Edition Article-I.

20. GALLAHUE, D. OZMUN, J. 2005. Compreendendo o desenvolvimento motor: bebês crianças, adolescentes e adultos. 3.ed. - São Paulo: Phorte. p.14-38.

21. GUZMÁN, C. 2003. Manual de criminalística, Buenos Aires: Ediciones La Rocca. p.90-110.

22. JOÃO, A.F; FILHO, J.F.; DANTAS, E. 1999. Seleção, orientação e detecção de talentos para ginástica olímpica feminina. Rev. Treinam. Desport. 4(1):7277.

23. JOÃO, A.; FERNANDES FILHO, J. 2002. Identificação do perfil genético, somatotípico e psicológico das atletas brasileiras de ginástica olímpica feminina de alta qualificação esportiva. Fitness \& Performance J. 1(2):12-19.

24. JUNIOR, A.; CUNHA, A.; SCHENEIDER, A.; DANTAS, E. 2006. Características dermatoglíicas, somatotípicas, psicológicas e fisiológicas da seleção brasileira feminina adulta de handebol. Fitness \& Performance J. 5(2):81-86.

25. LEIVA, J.; MELO, P.; GIL, M. 2011. Dermatoglifia dactilar, orientación y selección deportiva. REVCGJMC. 9(9):287-300. 
26. MAIA, J. 1999. Aspectos genéticos de la práctica deportiva: un estudio en gemelos. Rev. Paulista Ed. Fís. 2(13):160-176.

27. MARTÍNEZ LAGUNA, L.E.; TAMARIT MEDRANO, R.; RANGEL MAYOR, L. 2012. El empleo de marcadores genéticos en el proceso de selección de talentos. EFDeportes.com, Rev. Digital. Buenos Aires. 17(171). Disponible desde Internet en: http://www.efdeportes. com/efd171/marcadores-geneticos-en-seleccion-detalentos.htm (con acceso 019/01/2013).

28. NETO, G.; BAPTISTA, J. 2007. Desenhos Papilares e Inteligência Cinestésico-Corporal. Rev. do IGP. 3(3):4754.

29. NISHIOKA, G.A.C.; DANTAS, P.M.S.; FERNANDES FILHO, J. 2007. Perfil dermatoglífico, somatotípico y de las cualidades físicas básicas de los bailarines becarios del Centro de Movimiento Deborah Colker. Fitness \& Perform. J. 6(5):331.337.

30. NORTON, K.; OLDS, T. 2001. Morphological evolution of athletes over the 20th century: causes and consequences. Sports Med. 31(11):763-783.

31. RANKINEN, T.; BRAY, M.; HAGBERG, J.; PERUSSE, L.; ROTH, S.; WOLFARTH, B.; BOUCHARD, C. 2006. The human gene map for performance and healthrelated fitness phenotypes: The 2005 update. Med Sci Sports Exerc. 38(11):1863-1888.

32. RIBEIRO FERREIRA, H.; BARBOSA, F.; FERNANDES FILHO, J. 2008. Correlação entre níveis de preensão manual e dermatóglifos dos atletas da seletiva olímpica de canoagem slalom para Pequin 2008. EFdeportes.com, Revista Digital. Buenos Aires. 13(121). Disponible desde Internet en: http://www.efdeportes. com/efd121/seletiva-olimpica-de-canoagem-slalompara-pequin-2008.htm (con acceso 001/05/2013).
33. SKINNER, J. 2002. Será que a genética determina o campeão? Sports Science Exchange. Gssi.com.br, Gatorade Sports Sciences Institute n. 34. Disponible desde Internet: http://www.gssi.com.br/artigo/77 (con acceso: 28/04/2013).

34. TUCHE, W.; FAZOLO, E.; ASSIS, M.; DANTAS, E.; FERNANDES FILHO, J. 2005. Perfil dermatoglífico e somatotípico de ciclistas de alto rendimento do Brasil. Rev. Ed. Fís.132:14-19.

35. UNION CYCLISTE INTERNACIONALE UCI. 2013. Ranking Track. Disponible desde Internet: http://www. uci.ch/templates/BUILTIN-NOFRAMES/Template1/ layout.asp?Menuld $=$ MTYzNDE\&LangId $=1$ (com acceso 03/06/2013).

36. USA GYMNASTICS. 1998. Talent opportunity program manual. USA Gymnastics Federation - USA. p.1-2.

37. VOLKOV, V. 1983. Seleção esportiva. - Moscou: Educação Física e Esporte. p.176.

38. VONA, G.; MASSIDDA, M.; CIREDDU, M.; CALO, C. 2005. Genética e performance sportiva. Ital J. Sport Sci. 12(2):105-15.

39. WEINECK, J. 1991. Biologia do Esporte. São Paulo: Manole. p. 50-70.

40. WEINECK, J. 1999. Treinamento Ideal. São Paulo: Ed. Manole. p. 110-120.

41. ZAKHAROV, A. 2003. Ciência do treinamento desportivo. $2^{\text {a }}$ ed. Rio de Janeiro: Grupo Palestra. p.45-70.

Recibido: Junio 11 de 2013

Aceptado: Febrero 17 de 2014

\section{Como citar:}

Medellín, J.P. 2014. Caracterización dermatoglífica de las ciclistas colombianas de pista de altos logros en pruebas de semifondo. Rev. U.D.C.A Act. \& Div. Cient. 17(1): 45-52. 